\title{
THE GALL-BLADDER AS A SITE OF FOCAL SEPSIS.
}

\author{
By T. JOEKES, M.B. (Leiden), M.R.C.S., Pathologist.
}

It was in all probability the chance observation of a marked improvement in a case of "rheumatism" after tooth extraction which first drew the attention to the possibility of a causal relationship between some-often insignificant-focus of infection and other pathological manifestations in different parts of the body. Similar observations were afterwards made in respect to infections of the tonsils, naso-pharynx and the air sinuses. The interest of the profession in the problems of "focal infections" having been awakened, in quick succession the bronchi, the intestinal tract, the appendix, the kidneys, bladder, uterus, vagina, the prostate and the gall-bladder were recognised as possible seats or foci of infection, and it is now generally acknowledged that the problem of focal infection is a very important, albeit a very difficult one. In this chapter we are concerned with the gall-bladder as the site of focal infection.

One has always to remember that there are two factors to be considered in this problem of focal infection, namely, (a) the site of infection with its local signs and symptoms, and $(b)$ the type of infection. The latter is usually the more important factor.

Take, for instance, the example of a patient complaining of "rheumatism" in any of its manifold manifestations or localisations, be it called " fibrositis," " muscular rheumatism," " arthritis," " neuritis," " neuralgia," " sciatica " or " lumbago." It is rarely the case that some unmistakably specific cause can be indicted as responsible. Rarely will one be able to elicit say a gonococcal infection in the past history to guide one's further examinations, nor will one often experience such good fortune as mine after the Great War, when in six soldiers from Malta who all came complaining of rheumatic pains, mainly in the legs, a past history of bacillary dysentery some months previously was obtainable, in all of whom examination of their stools revealed the presence of dysentery bacilli. Appropriate treatment was at once instituted with the satisfactory result that the rheumatic complaints ceased soon after successful eradication of the intestinal infection.

In the majority of cases, however, one will not have the advantage of such direct hints as to how to proceed. Then it is that one has seriously to consider the possibility of some comparatively occult infected focus. In some instances one may obtain useful information from carefully recorded temperature charts, although in many cases the temperature tends to be sub-normal rather than raised. Increased sedimentation rate of the red blood corpuscles and a slight polymorphonuclear leucocytosis, particularly if there is also some toxic granulation of the polymorphs, are useful indications that such sepsis is present.

Having accepted the probable existence of an infected focus, our next task will be to identify that focus together with the infecting micro-organisms. Now I think it is generally accepted that a streptococcus is probably the most common cause of " rheumatism" in such cases, and although the toxic effects may emanate from a root abscess, a tonsil or a sinus, the colon, the prostate or the gall-bladder, the consequent symptoms may be very much the same. Successful treatment is largely dependant upon identification of its source with a view to eradication. If 
patients with " rheumatic" complaints also complain of toothache, repeated sore throats or a tender antrum, of abdominal discomfort and slight diarrhœa, or of prostatic difficulties, our attention is, of course, directed to the localisation of the infected focus, although even in these cases, the possibility of still another infected focus must not be forgotten. In other cases the site of infection will be revealed by tenderness of a tooth on percussion, by the pus squeezed out from crypts of tonsils, or darkness of an antrum on transillumination, by the examination of the stool or of prostatic fluid obtained by massage of the prostate. In quite a number of cases, however, the focus of infection is "silent," and only painstaking and sometimes elaborate examinations and investigations can prove its existence. Although the gall-bladder has of late been proved to be one of the most important seats of focal infection, only too frequently it must be relegated to this last category. It is, therefore, of the utmost importance when searching for an obscure focus of infection, that one should distinctly think of the possibility of the gallbladder harbouring the infection.

The problem of the gall-bladder is considered under three heads :-

(1) the secondary signs and symptoms which are found more frequently to accompany infections of the gall-bladder than of most of the other organs,

(2) the signs, symptoms and differential diagnosis of cholecystitis, and

(3) the bacteriological, chemical and other methods of investigation which can now be applied.

\section{r. Secondary Signs and Symptoms.}

(a) Digestive disturbances, i.e. a feeling of fullness and abdominal pressure, particularly after fatty meals, which is usually felt in the upper half of the abdomen. Although it is usually stated that in cases of chronic cholecystitis there is, as a rule, an accompanying hypochlorhydria, in the majority of cases I have examined I have found a distinct hyperchlorhydria.

(b) Flatulence and eructation, which account for the feeling of abdominal pressure.

(c) A " bitter" taste in the mouth on waking in the morning.

(d) Attacks of pain in the precordial region which sometimes closely resemble attacks of angina pectoris or of coronary thrombosis.

I have under my care at the moment a male patient, aged 68 , who in the course of the last two or three years has had several attacks of pain in the heart region as well as attacks of giddiness which have actually caused him to fall down without, however, losing consciousness. These attacks of pain and of giddiness have sometimes synchronised, at other times they have occurred quite independently of one another. The cardiologists he has consulted on account of these pains have never found anything radically wrong with his heart. Lately he has also suffered from attacks of sharp pain in the right upper quadrant of the abdomen which suggested gallstone colic, and X-ray examination showed the presence of stones in the gall-bladder. As he refused an operation, it was decided to see what could be achieved by non-surgical drainage of the gall-bladder by duodenal intubation. The gall-bladder was then found to be heavily infected with streptococci. Treatment was begun with regular duodenal drainages and the administration of olive oil in the morning. After the first two or three drainages the patient 
was greatly disturbed the following day by attacks of pain in the heart region and of giddiness. These were, no doubt, due to absorption of large amounts of heavily infected bile, from the gall-bladder, which had escaped into the duodenum and which it had not been possible to remove by the tube. However, he made steady progress and reported that he could feel the stones " move," and on being requested to look for stones in his motions whenever he thought that they had " moved" again, he did eventually find some. After the patient had made sufficient progress, it was decided to supplement the treatment with injections of an autogenous vaccine. After having lived the life of an invalid for two or three years, he now leads a very active life and is doing some very useful and important war work, and he has been free from attacks of either pain or giddiness for many weeks, except on one occasion, the day after a vaccine injection, when apparently too large a dose had been given. In consequence the patient not only had a general reaction, i.e. some rise of temperature, general malaise and headache, but he also again had some pain and giddiness, all of which would seem conclusively to prove the intimate connection between the infection of the gall-bladder and his original complaints.

\section{The Signs, Symptoms and Differential Diagnosis of Cholecystitis.}

Cases of acute cholecystitis, of either so-called " catarrhal " or " suppurative" type, with the well-known symptoms of sudden onset, high temperature and rigors, vomiting and acute pain in the gall-bladder region and great tenderness and marked rigidity of the right upper rectus muscle, do not fall within the scope of this article in which only examples of chronic inflammation of the gall-bladder merit our attention.

Briefly stated, the symptoms differ from those of acute cholecystitis above enumerated, by their comparative mildness, and the absence of high temperatures. Moreover, whereas in the acute forms the whole clinical picture is dominated by the manifestations of acute inflammation, in the chronic form the secondary symptoms such as indigestion, flatulence and belching very often inconvenience the patients more than the dull aching recurring pain in the upper part of the abdomen. This pain, however, varies greatly in character, localisation and severity, not only in different patients but often in the same patient from day to day, particularly when stones are present. The classical text-book picture of gallstone colic with severe pain in the right hypochondrium, radiating along the costal margin to the right scapula or right shoulder, though familiar to us all, is by no means always evident. Nor are any of the above mentioned symptoms sufficiently characteristic of gall-bladder disease to be "diagnostic"; they are to be found in other conditions.

For the purpose of differential diagnosis it is convenient to divide cases of cholecystitis in two groups, A. those without, and B. those with stones.

A. Cholecystitis. without stones. Bearing in mind the symptoms of chronic cholecystitis as briefly described above, difficulties may arise in differentiation from cases of

(a) Gastric ulcer. Here the pain is usually more directly related to a meal rich in proteins than in greasy food as is the case in gall-bladder disease: the pain also occurs at more regular intervals after food. In gastric ulcer, too, the pain is frequently relieved by alkalis, by the ingestion of milk and by vomiting. 
(b) Duodenal ulcer. The interval between the taking of food and the pain is usually somewhat longer, and the patient often experiences characteristic attacks of pain in the middle of the night.

In both (a) and (b) one can often demonstrate the presence of occult blood in the fæces, and X-ray examination will, in the great majority of cases, reveal the presence of an ulcer.

(c) Enteritis and colitis. A complaint of dull aching pain in the abdomen is frequent, but careful investigation will reveal that in enteritis the pain is often situated more in the middle of the abdomen and is often described as shifting about with little cramps : in colitis the colon is frequently found to be palpable and tender. Sufferers often state that bowel-movement or hyperperistalsis after taking laxatives has a definite influence on the pain. Examination of the fæces will often help to support the diagnosis in these cases.

From the above short summary it will be seen that the differentiation between cholecystitis and any of the conditions mentioned under (a), (b) or (c) is comparatively easy provided the possibility of any of these conditions is borne in mind.

(d) Chronic appendicitis. The subjective complaints are often very similar to those in chronic cholecystitis, although in most cases the differentiation between the two does not present too great difficulties.

B. Cholecystitis with cholelithiasis. The complaints are often very similar to those in:-

(a) Right-sided renal calculus, although in a left-sided renal colic the patient may occasionally complain of pain in the right side, and vice-versa.

(b) Hydronephrosis.

(c) Pyonephrosis.

In all these cases there will be more or less marked urinary symptoms. The pain may be described as radiating into the bladder region or even as far down as the tip of the penis. Large quantities of urine are often passed after the attack, and the urine may contain red blood cells or pus cells in varying amounts. X-ray examination, and particularly a pyelogram, will assist in the diagnosis.

(d) Arthritis or caries of the spine. Pain on movement or pressure locally, and an X-ray examination should reveal this condition.

(e) Gastric and other abdominal crises in tabes. This may offer great difficulties unless one takes a careful history of the case and examines for other manifestations of specific infection. Serological examination often clinches the diagnosis.

(f) Diaphragmatic hernia may produce symptoms of dyspepsia and pain very similar to those in cholecystitis. Here again an X-ray examination is conclusive.

(g) Pancreatic stone. Although in this condition the pain often radiates to the left and symptoms of chronic pancreatitis are usually present, differentiation from chronic cholecystitis with stones may be extremely difficult, or even impossible inasmuch as both conditions may be present at the same time.

(h) Many cases have been described in the last few years where gall-bladder diseases give rise to symptoms suggestive of coronary disease of the heart as in the patient described above, but there is often the typical relation between pain and 
exercise or emotional disturbance. The electrocardiogram will, in the majority of cases, show the characteristic changes. A few cases have been recorded with intense pain under the maubrium sterni which had been diagnosed as aortic aneurysm until an X-ray examination showed a normal aorta.

(i) Allergic persons with abdominal manifestations may sometimes offer very great difficulties in diagnosis. Some five or six years ago a healthy, sturdy boy of I5 was one day suddenly seized with violent abdominal pain soon after his dinner. He vomited copiously and also had diarrhœa. On examination the pain seemed to be located in the right half of the abdomen, and the right rectus muscle was contracted and "on guard." Although the boy was not of the complaining type, he could hardly be touched on account of the pain and tenderness, which appeared to be most severe over the region of the gall-bladder and the appendix. A provisional diagnosis of either acute cholecystitis or acute appendicitis was made with decision to operate immediately. A delay, unavoidable but in the circumstances fortunate, justified an alteration of opinion, for in the meantime the boy was apparently quite normal again, except that he felt very tired and, as he expressed it himself, "as if he had had a rattling good beating." In view of the rapid recovery and complete absence of any physical signs, the diagnosis was altered to " probably renal colic," and complete recovery having occurred next day, it was assumed that he had passed the stone. Some weeks later he had a similar attack. Many investigations yielded negative results, but a blood count revealed eosinophilia ( $I 7$ per cent.), and some mucus in the fæces was also found to contain numerous eosinophiles. It then transpired that the boy had a pronounced allergic family history although the patient himself had never had any allergic manifestations before. As both attacks had occurred half an hour after his dinner, the character of his food was carefully scrutinised, and it was then ascertained that on both occasions the boy had partaken of sweetbread. This has, of course, been carefully avoided ever since, and he has had no more attacks. This is, admittedly, an extreme case, but much less severe examples, some of which may amount to only slight discomfort after meals, are not very rare allergic occurrences.

These then are examples of the more commonly encountered manifestations of a wide variety of conditions in which gall-bladder disease-with or without stones-must be considered in the differential diagnosis. From these examples it is evident that in some cases one will only arrive at the correct diagnosis after a very careful process of elimination. In others it will be comparatively easy such as an easily-palpable tender gall-bladder, guarded by a slightly rigid right rectus muscle. Murphy's sign, when clearly present, is of great diagnostic value, i.e. on palpation of the gall-bladder region, the patient may experience a sudden pain on taking a deep breath, and the breathing may be suddenly checked. This sign may be best elicited if the patient is examined standing up and bending slightly forward.

What other means have we at our disposal to arrive at a definite conclusion?

(I) A point which perhaps does not quite fit in under the heading but which should be stressed as of the greatest importance, is a very careful history as to the origin and development of the symptoms, thus, relationship to food (both as regards the time factor and the character of the food), to physical exercises, emotions, sudden changes of temperature and the ways and means which the patient has found useful in alleviating the symptoms. 
(2) Cholecystography. Although this is perhaps the most useful single procedure in the diagnosis of diseases of the gall-bladder, particularly in the presence of stones, there are still many pitfalls and there is no justification for the attitude which nearly amounts to a conditioned reflex, to demand as a routine a cholecystogram as soon as the gall-bladder is suspected. It should always be remembered that cholecystography can only inform us whether the gall-bladder does or does not fill and empty regularly, and in those cases in which the dye does get into the gall-bladder, whether or not stones are present. In order that the dye can enter the gall-bladder, the cystic duct must be patent, so that in those cases where the duct is either blocked by a stone or kinked as a result of old adhesions, or where its lumen has been considerably narrowed as in "catarrh" of its lining mucous membrane, no dye will reach the gall-bladder and consequently no gall-bladder shadow will be visible. Very occasionally, too, it happens that a certain patient does not absorb the dye at all if given by mouth and that it simply passes through the intestinal tract. In such a case intravenous administration of the dye could be employed. Although, therefore, the absence of a gall-bladder shadow does inform us that the dye did not enter the gall-bladder, it does not explain why. The greatest difficulty, however, and the most deceptive one is that a diseased gall-bladder is on occasion quite capable of producing a normal cholecystographic shadow. So if there is no shadow at all, or if the $\mathrm{X}$-ray shows a normal shadow in these cases in which, on clinical grounds, one suspects a diseased gall-bladder, other methods of diagnosis will have to be considered, and

(3) Gall-bladder drainage by duodenal intubation is then indicated. In those cases which showed a normal shadow, in other words, in those in which the gall-bladder fills and empties normally, there will be an easy flow of bile after the instillation of magnesium sulphate into the duodenum. Even in a number of those cases where there was no shadow at all, it is often possible to empty the gall-bladder of its contents by gentle pressure over the gall-bladder after the magnesium sulphate has been introduced, particularly in those cases where there is a good responsive contraction of the gall-bladder, and one can feel the contracted gall-bladder. Apparently the added external pressure enables the contracting gall-bladder to overcome the obstruction in the cystic duct. Examination of the bile collected through the tube enables us to diagnose (a) whether or not there are stones present. When present, the bile always contains numerous cholesterol crystals which are either absent, or present only in small numbers, in the absence of stones, (b) which type of cholecystitis one is dealing with, i.e. an inflammation of the mucous membrane or mainly of the bladder wall (intramural infection). In the former case the bile contains numerous desquamated epithelial cells and pus cells, whereas in the latter, the pus cells are present only in small numbers, (c) the nature of the infecting micro-organisms. It is commonly stated that members of the coliform group of organisms are the most frequent offenders, but in a fairly large series of duodenal drainages, it has been my experience that streptococci are by far the most commonly encountered. Other organisms found come in the following order of frequency:-bacillus coli communis, the typhoid-paratyphoid and Salmonella group; bacillus Gærtner, bacillus Aertryche, bacillus Newport, staphylococci and occasionally Lamblia intestinalis.

It is of interest at this point to consider the various routes by which the micro-organisms reach the gall-bladder. Through the blood stream, i.e. by way of the cystic artery as well as along the portal vein, the lymphatics, and the bile duct. Bacteria may, apparently, travel from the duodenum against the bile 
stream into the gall-bladder. This commonly accepted route for typhoid bacilli seems to me highly improbable, as even in well-developed cases of typhoid the duodenum is usually not infected. It would appear to be much more probable that the infestation of the gall-bladder happens either along the arterial route during the early bacillæmia in typhoid, or along the portal vein from the infected bowel in the later stages of the disease. As a matter of fact, a normal healthy sphincter of Oddi is reputed not to admit anything, be it food, fluid or microorganisms. However, until we know more of the life-history of the Lamblia intestinalis - or Guardia lamblia, according to the new nomenclature-we must assume that they do reach the gall-bladder by swimming against the bile stream, but in this infection we know that the Lamblia is sometimes found in enormous numbers in the upper parts of the intestinal tract, right into the duodenum, and that they can be the cause of very debilitating diarrhoea. In some quarters it has been strongly decried that Lamblia can ever cause an infection of the gall-bladder and bile ducts, but in two of the patients in whom I had found numerous Lamblia in the duodenal fluid, I first washed the duodenum repeatedly until the collected fluid was almost free from Lamblia, before instilling the magnesium sulphate. In the bile collected afterwards they were again present in very large numbers. As, unfortunately, in both cases there was also a streptococcal infection of the gall-bladder, definite proof is still lacking whether the Lamblia actually did produce an infection or simply existed as accidental parasites in an already infected gall-bladder, or, lastly, whether there was an active symbiosis between those two micro-organisms.

With regard to infection of the gall-bladder by way of the portal vein, it is very likely that at any rate most of the micro-organisms of the large coliform group reach it along that route, whereas streptococci and staphylococci probably get there by the arterial route. In a number of cases I have been able to demonstrate the presence of the same type of streptococci in the gall-bladder as in a tooth-abscess, or in the naso-pharynx, or tonsils, in people who were subject to chronic sore throats and tonsillitis. This brings out a very important point in the whole subject of focal infection because although these patients sought medical advice for their complaints resulting from the cholecystitis,. it is more than likely that the infection of the gall-bladder was secondary to a similar infection either in the tooth-abscess, naso-pharynx or tonsil. This should warn one never to rest satisfied with the discovery of one infected focus, but to look for other foci from which the one already found may have been infected, or which may have arisen from it by dissemination.

Infection by the lymphatic route usually occurs from adjacent organs such as the liver, pancreas, etc.

Laboratory examination. Apart from the examinations which can be performed on the bile collected by duodenal intubation, there are no laboratory tests which are diagnostic for purely gall-bladder diseases. As, however, in nearly all chronic diseases of the gall-bladder the liver has been affected in some degree, help may be derived for the diagnosis by the liver function tests of which a great number are at our disposal. They are particularly useful inasmuch as they give information about liver damage before this is revealed by clinical evidence. Briefly summarised, the more common consist of :-

(a) The detection of the presence of bile and bile pigments in the urine.

(b) The presence of urobilin and urobilinogen in the urine. 
(c) Tests for bilirubin in the serum.

These tests not only aid in detecting early jaundice, not yet evident clinically, but also in differentiating between the three main types, viz. obstructive, toxic and hæmolytic jaundice (Van den Bergh's test).

(d) Determination of the icterus index.

(e) The lævulose and galactose tolerance test.

(f) Test for estimation of cholesterol in the blood.

\section{Treatment.}

As acute cholecystitis does not fall within the scope of a paper dealing with the gall-bladder as the site of focal infection, we are concerned only with the treatment of chronic cholecystitis. This falls into two headings, (a) the presence, (b) the absence of stones.

(a) Cases of chronic cholecystitis with stones should, generally speaking, be operated upon. It is of the utmost importance that the operation should be performed before complications such as extensive adhesions or perforations have occurred, or before the patient's general health has deteriorated so much as to render him a poor surgical risk, not only from the point of life or death but also from "after-operation complications." The operation of choice has, up to the present, been cholecystectomy. Time and experience will show whether Thorek's operation (electro-surgical obliteration of the gall-bladder without drainage), for which it is claimed that the mortality is only 0.5 per cent. as against 2 per cent. in cholecystectomy, and the post-operative discomfort is no greater than that after a simple appendicectomy, will in future become the standard operation.

There will always be, however, a number of patients who are too bad a surgical risk to justify surgical intervention or who are unwilling to undergo an operation, and for these medical treatment, including a series of duodenal drainages, should be instituted. Several recorded good results have conclusively refuted the dictum that " medical treatment of gallstones is a myth."

(b) Medical treatment. The early recognition of this condition is of prime importance. With all the diagnostic aids now at our disposal, as outlined above, there is no need to wait for the development of complications to enable us to arrive at the correct diagnosis, and the treatment should be dictated by our better knowledge and fuller understanding of the normal functions of the gall-bladder which are the storage and concentration of bile between meals and the expulsion of bile in response to the requirement of digestion. In infections of the gall-bladder its capacity to concentrate the bile is reduced or entirely lost, proportionately to the degree to which the mucous membrane has been affected. The normal chemical composition of the bile is altered thus diminishing its usefulness in the process of digestion, and the muscular contractability of the wall is impaired. Our aim, then, should be (a) to promote evacuation of the gall-bladder, thus eliminating its abnormal and infected contents and (b) carefully and gradually to re-establish the normal contraction of the gall-bladder. This can be done by the administration of a sufficiency - but not an excess - of fat and meat. Cream, butter and milk are excellent stimulants for the gall-bladder, but fried food which often produces pain and indigestion in people with cholecystitis should not be given, and in order to avoid too heavy a strain on the biliary system, five smaller meals per day are preferable to three larger ones. Among other substances known to be good 
stimulants for the gall-bladder are olive oil, magnesium sulphate, and peptone. Two tablespoons of the olive oil, or one or two teaspoonsful of magnesium sulphate in half a tumbler of water, should be taken first thing in the morning before rising, after which the patient should then lie on his right side for $15-20$ minutes. Of the magnesium sulphate so much should be given as to ensure one or two evacuations, but no diarrhœa. Ago-cholin, which is a useful combination of magnesium sulphate and peptone, may also be given in one or two teaspoonsful doses dissolved in water. In some cases in which it is not advisable unduly to stimulate the contraction of the gall-bladder, bile salts or sodium-di-hydrochloride, or an occasional dose of calomel should be given to produce the flow of a thin bile without contraction of the bladder wall.

For a more powerful and more complete evacuation of the bile a series of duodenal drainages should be instituted during which any of the above stimulants can be instilled directly into the duodenum. This method has the additional advantage of removing the infected bile and of preventing it from remaining in the intestinal tract and possibly infecting the bowels. The bile collected through the tube can also be examined and the nature of the infecting micro-organisms ascertained with a view to obtaining a culture and preparing a vaccine. Treatment with an autogenous vaccine has been found extremely useful in suitable cases, but its administration should be undertaken cautiously to avoid marked reactions. In some cases I have found a more ready response to the intracutaneous than to the subcutaneous administration of the vaccine. French authors claim excellent results by the oral administration of vaccine, especially in infections with any of the coliform group of bacilli. Lastly, one has to consider treatment witho antiseptics, some of which are excreted in the bile, enter the gall-bladder and then exert their anti-bacterial properties. The drugs most commonly used for thiso purpose are hexamine, salicylates, salyrgan and tetra-iodophthalein. As it is necessary to give big doses in order to obtain the desired result, and as all of them are apt to produce indigestion when given in such dosages, it is recommended to give big doses of sodium bicarbonate at the same time. Considerable difference of opinion still exists, however, with regard to the value of this antiseptic treatment.

Summary: Description of the different signs, symptoms and complaints which may arise from a focal infection in the gall-bladder with a discussion on the diagnosis, differential diagnosis and treatment of chronic cholecystitis. 